\section{Molecular Imaging: Fundamentals and Applications}

\author{
J. Tian, Ed.
}

New York, NY: Springer, 2013, 699 pages, \$279

This book is part of the series entitled Advanced Topics in Science and Technology in China, aimed at presenting the most up-to-date and cutting-edge theories, techniques, and methodologies in various research fields in China. The series is jointly published by Springer and Zhejiang University Press. The editor of this book and his coauthors are Chinese professors and scholars with various backgrounds, which include automation, electronic engineering, medical physics, chemistry, medicine, and life science.

I started my review of this book with great anticipation and found it particularly interesting because it focuses on effective information acquisition and processing methodology for biomedical studies. There are 3 parts with 20 chapters. The first part (chapters 1-11) presents fundamentals on molecular imaging theory and on the different imaging modalities, including diffuse optical tomography, fluorescence molecular tomography, bioluminescence tomography, PET, SPECT, MR imaging, and others. The introduction covers the development of molecular imaging and summarizes the basic principles, advantages, and disadvantages of each imaging modality. Chapter 2 focuses on the molecular optical simulation environment as it relates to the simulation of light propagation both in tissues with complicated shapes and in free space based on the Monte Carlo method. The chapters on diffuse optical tomography, PET, and medical image processing and analysis (chapters 3,6 , and 11 , respectively) contribute more detailed theories and methodologies than the chapters on fluorescence, bioluminescence, SPECT, MR imaging, and other modalities. The second part (chapters 12-15) covers the opportunities and challenges of radiolabeled probes, oligonucleotide probes, quantum dots, and others. The last part (chapters 16-20) illustrates mainly the basics of molecular biology and molecular imaging applications in preclinical and clinical research-for instance, clinical practice for tumors, protein-protein interactions, transgenic animals, and diabetes-related studies. Overall, through discussing the problems and challenges in detail and illustrating recent progress and future directions, this book covers novel theories and algorithms, the latest molecular probes, cutting-edge imaging systems and typical experiments, and the preclinical and clinical applications of recent years.

Over the past few years, molecular imaging has become a rapidly developing and promising multidisciplinary research area that has attracted significant attention in preclinical research on cancer theranostics and drug development. This book is best suited for researchers and postgraduate students in the fields of radiology, nuclear medicine, imaging physics, and computer science and other individuals who are interested in working on in vivo molecular imaging.

Mei Tian

The Second Hospital of Zhejiang University School of Medicine 88 Jiefang Rd. Hangzhou, Zhejiang 310009, China E-mail:meitian@zju.edu.cn

Published online Jan. 8, 2015

DOI: 10.2967/jnumed.114.153353

\title{
Erratum
}

Two articles inadvertently omitted acknowledgment of support by UC Discovery Grant 129978: "Novel Molecular Probes for Noninvasive Imaging." The two articles are "Novel PET Probes Specific for Deoxycytidine Kinase" by Shu et al. ( $\mathrm{Nucl} \mathrm{Med.} \mathrm{2010;51:1092-1098)} \mathrm{and} \mathrm{"Stratification} \mathrm{of} \mathrm{Nucleoside} \mathrm{Analog} \mathrm{Chemotherapy} \mathrm{Using}$ 1-(2'-Deoxy-2'-18 F-Fluoro- $\beta$-D-Arabinofuranosyl)Cytosine and 1-(2'-Deoxy-2' - ${ }^{18}$ F-Fluoro- $\beta$-L-Arabinofuranosyl)5-Methylcytosine PET" by Lee et al. (J Nucl Med. 2012;53:275-280). The authors regret the error. 